\title{
Per una grammatica degli errori di studenti catalani in italiano come L2
}

\author{
Gabriella Gavagnin
}

\begin{abstract}
Abstrasct
This paper catalogues the linguistic errors made by some $C$ atalan students in spontaneous written productions in Italian and tries to elaborate an interpretative hipothesis about heir frequency and distribution, especially referring to the processes of transfer from L1.
\end{abstract}

\section{Premessa}

II presente studio, basato sull'analisi di una serie di elaborati di studenti universitari catalani svolti in momenti diversi ma nelle stesse condizioni, cioè in sede di esame finale di corsi di linguistica o di letteratura, ${ }^{1}$ ha lo scopo di individuare una tipologia di errori e di fornirne ipotesi interpretative in funzione della loro frequenza e distribuzione. $N$ aturalmente sono significative soprattutto quelle deviazioni che si riscontrano nel maggior numero di studenti, in quanto spie di un aspetto problematico. N elle tabelle finali ho raggruppato gli errori del materiale spogliato (29 esami svolti da 22 studenti) per settori grammaticali, indicando la loro provenienza con il numero assegnato a ciascun elaborato. ${ }^{2} \mathrm{H}$ o preso in considerazione tutti gli errori di tipo grafico-fonetico, morfosintattico e lessicale (non sono segnalate eventuali ripetizioni dell'errore nello stesso elaborato), ma non ho tenuto conto (salvo alcuni casi di deviazioni stilistiche riguardanti l'ordine delle parole) degli aspetti inerenti l'organizza-

1. La preoccupazione dello studente è perciò rivolta più ai contenuti che non alla lingua. Inoltre, gli esaminandi non potevano consultare né vocabolari né libri. Non bisogna dimenticare, tra l'altro, che il loro metodo di studio abituale si basa sulla memorizzazione degli appunti presi a lezione, magari integrati da altre letture, ma comunque considerati punto di riferimento prioritario. II materiale linguistico degli esami è costituito in maggior parte dalla lingua del professore trascritta frettolosamente durante le lezioni e ristrutturata poi nella memoria dello studente. Esiste quindi un filtro fonetico da non sottovalutare in questi esempi di lingua scritta. N e è prova la presenza isolata di alcune grafie devianti che trascrivono pronunce regionali, in questo caso meridionali, dell'italiano, la cui origine risale al docente (introdusce, abbita, dubbita, cortiggiano, prestiggio).

2. O ccorre precisare che adotto per comodità l'etichetta di errore pur includendo usi devianti dello standard non classificabili esattamente come erronei (come le mancate elisioni di taIuni articoli o determinatescelte preposizionali), ma interpretabili come tali nel quadro delle conoscenze linguistico-grammaticali dei soggetti esaminati. 
zione sintattica del discorso. T uttavia, ho usato anche la testualità come parametro per stabilire approssimativamente il livello di ciascun testo considerato, in una scala di valori che oscilla da A a D e che tiene conto solo della competenza scritta. ${ }^{3}$ I dati suggeriscono anche quali sono all'interno di ciascun settore gli aspetti più esposti all'errore (per esempio le consonanti doppie nella grafia e le preposizioni nella morfosintassi). O vviamente, alcuni settori sono rappresentati in modo scarso nel tipo di testo preso in esame; per esempio, si osserverà che gli errori nell'uso di deittici e di determinati pronomi personali sono quantitativamente irrisori, perché, analogamente ad altre strutture più tipiche del parlato, sono poco ricorrenti nel discorso scritto.

\section{G rafia}

II settore della grafia con il maggior numero di errori è quello delle consonanti doppie, soprattutto per l'alta frequenza con cui esse ricorrono in italiano, sebbene, proprio in rapporto a tale frequenza, le deviazioni siano relativamente contenute. Infatti, i processi analogici, e quindi i transfer positivi dalla propria lingua, possono favorire il corretto impiego delle doppie in italiano. Per esempio, in corrispondenza dei nessi castigliani o catalani ct e pt gli studenti tendono a usare una doppia t, con risultati, in generale, corretti; così è normale trovare prodotto, atto, attuare, conflitto, concetto ecc. II ragionamento può di rado provocare raddoppiamenti inesistenti in italiano (così in prattici modellato su práctico), o può indurre in scempiamenti inopportuni qualora il castigliano abbia perduto anticamente il nesso di orientamento (così in soggeto 0 in scritura), ma più spesso costituisce il riferimento più immediato ed efficace per orientarsi nella selva delle doppie. In questo caso, il ricorso alla L 1 aiuterà senz'altro lo studente e mi sembra oltremodo utile stimolare in lui una riflessione cosciente su una serie di semplici meccanismi analogici che possono essere applicati con un successo molto elevato. Insomma, il ruolo dell'interferenza, sia positivo che negativo, non va sottovalutato neanche nel caso delle consonanti doppie, considerate di solito un problema interno della struttura fonologica dell'italiano e svincolato dai tipici processi di confronto e incrocio tra L 1 eL 2 .

D alla lettura statistica dei dati spogliati si può appurare innanzitutto che le occorrenze di raddoppiamento sono superiori a quelle di scempiamento improprio (65 contro 50). Anzi, se prescindiamo dai casi di degeminazioni in parole che hanno una sequenza di doppie, la tendenza al raddoppiamento assume dimensioni molto significative (65 contro 29). N on tutte le consonanti presentano gli stessi rischi: dal quadro riassuntivo, articolato per fonemi rispettivamente sonori e sordi, si desume che alcune consonanti risultano più problematiche di altre, il che può anche essere in rapporto alla loro frequenza nella struttura fonologica dell'italiano (la doppia v, per esempio, ha una fre-

3. La maggior parte di elaborati sono di livello medio-alto, poiché si tratta di studenti fre quentanti il secondo ciclo del corso di laurea in Filologia Italiana. 
quenza bassissima rispetto alla doppia I o alla doppia p). In particolare, nel nostro schema, le consonanti più colpite sono la t, la f e la $\mathrm{m}$, e in misura leggermente inferiore la s e la p. Per quanto riguarda la t, il raddoppiamento si verifica soprattutto nel segmento finale -atto/a/i/e (7 su 13), forse per attrazione dei participi passati con raddoppiamento. Si potrebbe interpretare in modo analogo sia il caso della $\mathrm{f}$, dove la frequenza dell'errore si concentra nel segmento iniziale deff- / diff- (9 su 11), rendendo plausibile l'ipotesi di un raddoppiamento analogico su forme molto comuni come differente o difficile, sia quello della $m$, dove più della metà di geminazioni scorrette coinvolge il segmento iniziale comm-, ma qui potrebbero influire i processi di interferenza con altre lingue (le parole interessate presentano una doppia in quelle corrispondenti in francese 0 inglese) o l'attrazione esercitata da al tre parole ital iane di uso comune (commercio, commedia). Infine, le oscillazioni nell'uso della s potrebbero collegarsi anche all'alternanza nel sistema grafico catalano tra s/ss per differenziare la sonora dalla sorda, mentre molti scempiamenti (per esempio nel caso della p) figurano in parole con un'elevata somiglianza formale con le corrispondenti forme castigliane e catalane: apare (aparece/apareix), aparizione (aparición/aparició), aprezzato (apreciado/apreciat), oposti (opuestos) oposats), oposizione (oposición/oposició), presupone (cast. presupone).

Gli altri fenomeni devianti della grafia sono di origine fonetica, mentre sono del tutto irrilevanti gli errori relativi all'uso di digrammi e trigrammi (più frequenti magari a livelli di competenza più bassi). Poiché, come noto e come confermano i dati di questo spoglio, la fonetica è tra i settori più esposti alle interferenze, possiamo considerare i tratti grafici seguenti come tipici di studenti ispanofoni e catalanofoni:

a) Confusione tra la bilabiale e la labiodentale (b/v).

b) Oscillazioni nell'uso della e davanti a nessi composti da s implicata aggiunta (especialmente, l'especulazione, espirituale) o soppressa per ipercorrettismo (stranea, spressivo). L'abitudine fonetica ad evitare tali nessi in posizione iniziale è testimoniata dalla frequenza con cui essi sono preceduti dalla forma dell'articolo o del dimostrativo non collocatile foneticamente in tali contesti: del studio, quel stimolo, un strumento, quel studio, nel stesso, sintagmi dietro cui si cela la tendenza della studente, nel momento in cui scrive, a pronunciare mentalmente tali forme con una e o una i prostetica.

c) M ancata elisione dell'articolo femminile indeterminativo e, davanti a i (per influsso del catalano), di quello determinativo.

\section{M orfosintassi}

N ella morfologia un settore particolarmente colpito è quello dei plurali femminili, sottoposti a un processo di semplicazione e di regolarizzazione dei paradigmi. L'allineamento delle desinenze di nome e determinanti avviene indifferentemente in -i 0 in -e. 
II genere del sostantivo non costituisce un particolare problema (se si pensa alle difficoltà che presenta per studenti germanofoni per esempio), perché le differenze tra castigliano, catalano e italiano sono minime. In questo caso, l'interferenza agisce quindi positivamente. Tuttavia, le poche divergenze dalla $\mathrm{L} 1$ vengono di solito ignorate.

Riguardo ad aggettivi e pronomi, vale la pena segnalare la presenza diffusa del possessivo suo invece di loro, dovuta com'è ovvio a un meccanismo di transfer, e il problema delle costruzioni con il dimostrativo neutro, che rivela anche qui un processo di interferenza un po' più sofisticato: i due casi riportati traducono altrettanti costrutti castigliani, sostituendo al neutro lo il neutro italiano quello, secondo un'analogia molto spesso valida, ma in questo caso inopportuna. Le frasi, volendo, sono corrette grammaticalmente, ma assolutamente fuorvianti, giacché per evitare che quello possa essere interpretato come un maschile singolare lo si dovrebbe sostituire con il neutro ciò. L'altro impiego deviante del dimostrativo quello è anch'esso frutto di un'ingannevole analogia. II castigliano e il catalano usano regolarmente l'articolo con funzione dimostrativa davanti ad aggettivi o davanti a frasi preposizionali e a proposizioni relative, mentre l'italiano adopera di norma il dimostrativo quello, salvo davanti a possessivi o aggettivi che possono ammettere sia il pronome che l'articolo. Tuttavia, con i numerali, come in questo caso (quello secondo), il pronome dimostrativo è sempre sostituito dall'articolo, un'eccezione che sarebbe utile, in sede di riflessione grammaticale, pre sentare allo studente come tale. Infine, per gli indefiniti emerge il problema dell'asimmetria di numero nell'opposizione qualche / alcuni, assente nella L1.

Per quanto riguarda la morfologia verbale, il dato più significativo mi sembra sia il tipo di errore nelle desinenze: tranne qual che caso imputabile a processi di regolarizzazione del paradigma, si tratta in sostanza di forme modellate su quelle della L 1. Si pensi soprattutto a conceputo (cat. concebut) 0 prendrà (cat. prendrà), e a tenemo (cast. tenemos) o creamo (cast. creamos).

Interessante per osservare l'evoluzione degli errori a livelli di competenza diversi è il caso delle costruzioni con l'impersonale esserci. II calco lessicale caratterizza i livelli più bassi dove si riscontra avere per esserci, mentre, a un livello più alto, I'interferenza agisce più sottilmente sulla sintassi, con la neutralizzazione dell'opposizione singolare/plurale, tipica del castigliano e tendenziale in catalano.

Le preposizioni, com'è da aspettarsi, costituiscono una fonte perenne di errore a ogni livello di competenza. O stili a ogni tentativo di astrazione, esse sfuggono costantemente a tutti gli schemi con cui si cerca di ridurle a un ordine razionale, restando spesso in una zona di incerta definizione e descrizione. Tuttavia, dagli errori riscontrati in questi campioni, notiamo che almeno per il $50 \%$ si tratta di strutture relativamente controllabili dagli studenti. M i riferisco, in particolare, agli scambi tra di e da o tra per e da, dove cioè l'uso di da rientra in una regola generale, di tipo semantico in un caso (origine, provenienza, distacco, ecc.) e di tipo sintattico nell'altro (complemento d'agente). L'errore con maggiore incidenza è proprio il sottoutilizzo 
della preposizione da, avvertita come una forma estranea, non riconducibile a un'equivalente forma della $L 1$. In realtà, in questo settore è particolarmente potente l'azione di transfer dalla L1 alla L2 (è sintomatico il caso di a partire di, formula del resto molto meno frequente dal punto di vista stilistico in italiano). Analogo discorso si può fare anche per le locuzioni preposizionali, le congiunzioni e gli avverbi, dove le interferenze costituiscono la causa principale, se non addirittura unica, d'errore. Alcune deviazioni spiccano per la loro alta occorrenza, come nel caso della congiunzione subordinante mentre che invece di mentre. Gli errori possono essere di forma (fino invece di fino a, davanti invece di davanti a, attraverso di invece di attraverso, ancora più invece di ancor più: molto complessi in genere gli accumuli di preposizioni e avverbi) o di semantica (ogni volta più per sempre più, finalmente per alla fine, anche per inoltre, come per quanto).

Per quanto riguarda la sintassi verbale, i dati indicano chiaramente due costruzioni a rischio: le concessive (in cui si prescinde sistematicamente dal congiuntivo) e i gerundi, subordinanti o fraseologici, presenti in eccesso. $\mathrm{D}$ 'altronde, esse figurano tra le non molte divergenze tra castigliano, catal ano e italiano in questo settore.

Un altro aspetto su cui mi sembra necessario richiamare l'attenzione riguarda l'ordine delle parole nella frase, un aspetto tradizionalmente trascurato dalle grammatiche. In particolare, i numerosi enunciati devianti riportati coinvolgono un numero molto limitato di elementi, l'avverbio solo/soltanto e soprattutto le congiunzioni anche/neanche, il cui uso appare quindi decisamente problematico nella $L 2$ di studenti ispanofoni, tale almeno da meritare una maggiore attenzione in sede di riflessione grammaticale.

\section{Lessico}

II dato più macroscopico nell'ambito lessicale è il ruolo determinante svolto dai processi di transfer, i quali, sebbene palesati negativamente dagli errori, agiscono costantemente su tutta la materia lessicale grazie all'elevato grado di affinità tra la L1 e la L2, fattore da sfruttare senz'altro come strumento di apprendimento accelerato, ma da controllare con opportune strategie didattiche per le insidie che parallelamente esso comporta.

In un primo tentativo di raggruppamento dei termini devianti si possono prendere in considerazione tre variabili:

1. Se si tratta di ispanismi più o meno italianizzati, ma privi di un tipo lessicale somigliante in italiano;

2. Se la parola è un ibrido dal punto di vista formale tra il termine italiano e quello della L 1 ;

3. Se la deviazione non è formale ma semantica.

Sono cosi riuniti nel gruppo dei «Prestiti italianizzati» i pochi casi in cui il processo di italianizzazione di una parola castigliana o catalana è stato appli- 
cato su parole inesistenti in italiano. M olto più folte sono, invece, le liste dei calchi semantici e dei termini ibridi, risultato appunto dell'enorme somiglianza formale del lessico tra le tre lingue in questione. In particolare, gli ibridi permettono di analizzare anche quali elementi resistono maggiormente ai meccanismi di trasformazione del termine della $L 1$ in quello della $L 2$. Per esempio: difficilmente ci si dimentica della vocale finale nei morfemi verbali o nominali, come pure i nessi o fonemi consonantici estranei all'italiano sono di norma sempre sostituiti da nessi o fonemi esistenti in italiano (cast. e cat. conflictiva $\rightarrow$ conflittiva o cast. extraer $\rightarrow$ straerne), tranne qualche eccezione (fragmentazione) riscontrabile a livelli di competenza più bassi. Invece, la scelta delle vocali atone i / e (sollicitati, correspondenti, provinente) o dei suffissi (intervenzione, salvazione, evoluzionerà, conflittiva, passo, manca) appare molto problematica a tutti i livelli.

\section{Conclusioni}

Come si è cercato di mettere in luce in questa lettura generale dei dati classificati, il ruolo svolto dall'interferenza nella dinamica degli errori ha dimensioni notevoli e agisce secondo meccanismi attivati in genere in modo involontario dallo studente; talvolta, però, questi può ricorrere in modo cosciente alla $\mathrm{L} 1$ alla ricerca di analogie che lo aiutino a colmare lacune fonetiche o grammaticali. Invece, i fenomeni di semplificazione linguistica indipendenti dalle strutture della lingua propria hanno un'incidenza molto limitata soprattutto, ma non solo, a livelli medio-alti. I dati confermano che la L1 (anzi le L1, poiché in soggetti bilingui o diglossici possono verificarsi pressioni simultaneamente da entrambe le lingue possedute) interferisce soprattutto in settori come la sintassi e il lessico, come noto più esposti a tale fenomeno, e in misura minore, ma non per questo da sottovalutare, nella grafia e nella morfologia, dove emergono parallelamente anche processi di semplificazione linguistica ricorrenti nell'italiano di chi ha una diversa L 1. Per esempio, la tendenza alla regolarizzazione del paradigma di sostantivi e aggettivi femminili è ampiamente riscontrata anche nell'italiano popolare, cioè nell'italiano di dialettofoni. Infine, riguardo all'interferenza, i dati analizzati evidenziano che essa si manifesta sotto forma di meccanismi diversificati che andrebbero esplorati in modo più sistematico perché la loro conoscenza può senz' al tro migliorare eventuali strategie didattiche. 
Errori risultanti dallo spoglio di 29 elaborati di studenti catalani ${ }^{4}$

\section{Grafia-fonetica}

\section{Doppie}

b

abbitano 21.

dubbita 9. p

copprono 19. scoppo 20. d

cadde 3.

iddentificarsi 12.

g [velare]

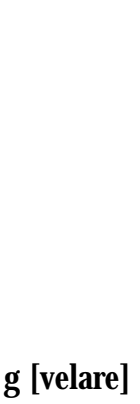

radoppiamento $14 \mathrm{~b}$. 15a.15b.16a.16b. 17b.20. $\mathbf{t}$

prattici 5.

datta 11 .

legatto 13.

collegatti 9.

amalatti 9 .

sostratto 15b.16b.

diastrattici 10.

cadutta 13.

percepitte 9 .

cattena 3.

rottacismo 15b.

prottegono 21.

\section{c [velare]}

ragruppamento $15 \mathrm{~b}$. riccupera 1 .

prottegono 21 dopodicché 8. grecco 18. oposizione 20.

oposti 8.

apare 4.

aparizione 13.18.

aprezzato 11a.

dopie 15a.

aprendimento 21 .

presupone 5 .

produtrice 5 .

legitimo $7 a$.

addiritura 8.

stretamente 9.

scritura 13.

maritime 15b.

soprattuto $15 \mathrm{~b}$.

monotonghe $17 \mathrm{a}$.

soggeto 21 . chiacherare 9.

oclusive 17a.

4. Riporto qui la descrizione di ciascun elaborato:

\begin{tabular}{|c|c|c|c|c|c|}
\hline $\begin{array}{l}\text { Num. } \\
1 \\
2 \\
3 \\
4 \\
5 \\
6 a \\
6 b \\
7 a \\
7 b \\
8 \\
9 \\
10 \\
11 a \\
11 b \\
12\end{array}$ & $\begin{array}{l}\text { D ata } \\
\text { II-92 } \\
\text { II-92 } \\
\text { II-92 } \\
\text { II-92 } \\
\text { XII-95 } \\
\text { VI-95 } \\
\text { XII-95 } \\
\text { VI-95 } \\
\text { XII-95 } \\
\text { XII-95 } \\
\text { VI-95 } \\
\text { VI-95 } \\
\text { VI-95 } \\
\text { II-96 } \\
\text { VI-95 }\end{array}$ & $\begin{array}{l}\text { Livello } \\
B \\
D \\
D \\
C / D \\
B / C \\
D \\
D \\
C / D \\
D \\
C / D \\
C \\
D \\
C / D \\
D \\
C / D\end{array}$ & $\begin{array}{l}N \text { um. } \\
13 \\
14 a \\
14 b \\
15 a \\
15 b \\
16 a \\
16 b \\
17 a \\
17 b \\
18 \\
19 \\
20 \\
21 \\
22\end{array}$ & $\begin{array}{l}\text { D ata } \\
\text { VI-95 } \\
\text { XII-95 } \\
\text { II-96 } \\
\text { XII-95 } \\
\text { II-96 } \\
\text { XII-95 } \\
\text { II-96 } \\
\text { XII-95 } \\
\text { II-96 } \\
\text { II-96 } \\
\text { II-95 } \\
\text { II-95 } \\
\text { VI-95 } \\
\text { VI-95 }\end{array}$ & $\begin{array}{l}\text { Livello } \\
\text { B/C } \\
\text { D } \\
\text { D } \\
\text { C/D } \\
\text { C/D } \\
\text { D } \\
\text { D } \\
\text { A/B } \\
\text { B } \\
\text { A/B } \\
\text { D } \\
\text { D } \\
\text { B/C } \\
\text { L1: italiano }\end{array}$ \\
\hline
\end{tabular}




\section{g [palatale]}

cortiggiano 11a.

prestiggio 13.

\section{$\mathbf{s}$}

bassi «basi» 8 . issoglosse 10.

criticandossi $11 \mathrm{~b}$.

assimetrico 16b. 20.

assimmetrico 19.

essempio 21.

rissale «spicca» 3.

m

communità 5 . commodo 5.

comminciare 9 .

communicare 13.

communicazioni $15 \mathrm{~b}$.

gemminazione $17 \mathrm{a}$.

degemminazione 14b.

arriveremmo 13.

parleremmo 11 a.

I

ellisione 14a.

sibillanti 14b.

\section{Sequenza di doppie}

efetto 20.

sufisso $14 \mathrm{~b}$.

fanciulezza 9.

soggeto 21 .

soprattuto $15 \mathrm{~b}$.

addiritura 8.

ragruppamento $15 \mathrm{~b}$.

\section{b/v}

riproveboli 9.

fabole 11a.

trobatori 11a.

servo-croata 21. f

deffiniamo 1.

deffinito 5 .

rifflettono $6 a$.

diffendendo $6 \mathrm{~b}$.

diffende 7a.7b.12.

diffenditori $7 \mathrm{~b}$.

deffine 9 .

diffesa 12.

riffare 13.

\section{c [palatale]}

sfugire 3. fiduccia 9

attegiamento 9. acenna 8.

faciamo 6b.

\section{z}

presione 19.

nesi 18.

conoscese 3.

concezzioni 7a.

attualizano $7 \mathrm{a}$.

razzionalizazione $7 a$. razzionalizazione $7 a$. adozzione 13. democratiza 13. venezziano 15b. caratterizanti 18. negozzi 21.

n

inimaginabile 11b. personnaggio 9. verrano 9. assimetrico $16 \mathrm{~b}$. divienne 13 . comentare 20. branno 18. 20. amalatti 9. sufisso 14b.

difusione $16 \mathrm{~b}$.

diferenza 18.

efetto 20.

\section{r}

fanciulezza 9.

dal'ignoto 9.

prerromana 10.

aprezzato 11a.

attualizano $7 \mathrm{a}$.

caratterizanti 18.

verrano 9.

acenna 8.

attegiamento 9.

radoppiamento 14b.15a.15b.16a.16b.17b.20.

diavolici 11a.

avvia «abbia» 13.

doviamo 21. 


\section{s implicata}

especialmente 20 . del studio $5 . \quad$ stranea $6 \mathrm{~b}$.

l'especulazione 7a. quel stimolo 7a. spressivo 13.

espirituale 8. un strumento 7a. straerne 4.

esperimentare 7a. quel studio 8.

nel stesso 13.

\section{Elisioni e troncamenti}

la Estetica 5.

la Italia 17b.

alla influenza $17 \mathrm{~b}$.

la impossibilità 19. una espressione $5 . \quad$ lo $\mathrm{H}$ egel 8.

una enorme 9.

una esperienza 9.

una identità 13.

una alternanza 17a.

una assimilazione 19.

una indicazione 20 .

una alternanza 20.

\section{Accenti}

e «è» 1.7a.13.15b.21.

ne «né» 20. pero «però» 1 . da «dà» 17a.

\section{D eglutinazioni e agglutinazioni}

su la 11a.

a la $16 a$.

a l'infinito 20.

a l'italiano 21.

\section{Altro}

spezializzate $6 a$. enunziati $6 a$.

fragmentazione $17 \mathrm{~b}$.

strictamente 14a.

introdusce 5. per ciò 15b.

già che 18 .

per tanto 11b.18. uno esercizio 9.

uno episodio 9.

uno insieme 9.

uno intellettuale 13.

dell'espressioni 5.

dell'interazioni $6 b$.

un'altro $7 b$.

un'amore 9.

macché «ma che» $11 a$.

\section{M orfosintassi}

\section{Plurali femminili}

due soluzione 1.

le arme 9.

le manifestazione scritte 13.

delle regione $16 \mathrm{~b}$.

alle assimilazione $16 \mathrm{~b}$.

le iniziale latine 16b.

\section{G enere del sostantivo}

arte costituito $6 \mathrm{~b}$.

la tentativa 5.

la paradossa $7 \mathrm{~b}$. archaiche 17a.

cerchare, ricercha 8.

traccie 18.

sporchizia 3. 


\section{Articoli}

i pseudoconcetti 5.

li strumenti 13. dei aspetti $17 \mathrm{~b}$.

nei altri 18. gli parlanti 21.

une forme $17 \mathrm{a}$.

\section{Pronomi personali e relativi}

\section{essa ... lei «ella ... ella» 2.}

a lei «ad essa» 4.

prendeloro «essi» $7 b$.

tra egli $17 \mathrm{~b}$.

prende questa noz. dal D e Sanctis, chi non tiene conto $6 \mathrm{~b}$.

cui: sogg. persona 11a.

\section{Possessivi}

suo/a/oi/e «oro» 7a.9.11a.16b.18.

\section{D imostrativi}

quello/ciò

Era, appunto, l'intervento della fortuna quello che poteva 2.

l'amore è quello che permette $7 \mathrm{a}$.

quello/il

il primo momento ... quello secondo 8.

il proprio Boccaccio «o stesso Boccaccio» 3.

\section{Indefiniti}

qualche linguistici 5.

alcuna grafia 19.

alcun altro caso 20.

tutta possibile... «ogni possibile» $7 \mathrm{a}$. si aspetterebbe «ci siં 19.

si aggiunge uno «sene... »1. tutte e due lingue $6 a$.

tutti e due registri 11a.

in tutte e due aree 19.

tutti i tre 13.

\section{Verbi}

\section{D esinenze:}

tenemo 5.

creamo $7 a$.

define $7 a$.

apparono 17a.

scompariscono 20.

prendrà 13.

sembrarebbe 3.

producerebbero $6 \mathrm{~b}$.

conceputo 6b.

dividita 20.

dividiti 21.

\section{Accordo participio}

affermatosi «affermatisi» $11 b$.

li ha imparato 21.
Avere/Essere:

aveva stato 5.

ha penetrato «è penetrato» $6 a$.

avevano esistito $11 b$.

hanno passato «sono passate» $16 \mathrm{~b}$.

Costruzioni impersonali:

C'è «ci sono» 19.

ha avuto «c'è stata» 18.

avere «esserci» 17a.

\section{Costruzioni riflessivo-pronominali}

evadersi «evadere» 9.

s'inizia «inizia» 12.13.

si è cambiata «è cambiata» 13 . 


\section{Preposizioni}

\section{$\mathrm{Di} / \mathrm{Da}$}

a partire di/del/... 7a.7b.8.9.13.

evadersi della 9.

allontanato di $7 \mathrm{a}$.

separata delle 5 .

si isola del $7 a$.

diverso/a/i/e di/del/... 5.7a.

dipendere di/del/... 10.16b.21.

proviene di $15 a$.

scompariscono dell'uso 20.

servira di base 12 .

\section{Per/Da}

viene deffinito per questo 5 .

prodotta per 13.

sostituita per 14b.

siano caratterizzati per $17 \mathrm{~b}$.

per un'altra parte $17 \mathrm{~b}$.

sfugire delle 3.

Iontano di 21.

si allontanano di/del/... 4.20.

separa di $17 \mathrm{~b}$.

slegata del 12.

non si distinguono di 5.

provinente di 13.

viene delle 17a.

accompagnate di 12 .

novella di caratteristiche picaresche 2 .

dominati per 13.

favorito per 21.

è caratterizzato da... e per 15b.

per una parte 17a.

per i linguisti «da parte dei» 5 .

\section{A/D a}

diverso/a a 8.21.

il modello a seguire 12.

\section{$\mathrm{Da} / \mathrm{Di}$}

più spiritoso da quelli 9.

stessa formazione dai siciliani 9.

il suo uso da questo autore 19.

è il prodotto da quello precedente 2 . esclusiva dalla 13.

\section{A accusativo}

al quale $7 a$.

influenzerà agli intellettuali 11b.

\section{In/Su}

tre punti in cui gira 3 .

nel limite superiore 8.

basato in/nel/... 11a.13

ripercussione nel 15a.

in un piano inferiore $6 \mathrm{~b}$.

nel piano fisico 9.

nei margini «sui, ai» 11 a.

\section{A/C on - C on/A}

collegamento alla «con la» 5.

in rapporto colle idee «alle» $7 \mathrm{~b}$.

si scontra a «con» $7 a$.

\section{Altri casi}

di molto piccolo 12.

previe delle cose «previe alle» $7 a$.

un sistema di cinque gradi «a cinque

gradi» 16b.

\section{Preposizioni rette da verbi}

per quanto riguarda $a / a l / \ldots 5.12 .20 .21$. diventa in «diventa» 3. conta con «su» $6 a$.

si ispirano in «a» 13.

ha sostituito x per $\mathrm{y}$ 《con» $14 \mathrm{a}$.

l'amore alla donna 7a.

sul periodo dei «nel periodo dei» 20.

permette $[. .$.$] elaborare «di elaborare» 6 b$.

traducono al «in» 12.

pensare in «a» 21.

ha sostituito da «con» 19.

accennare l'uso «a» 15. 


\section{Preposizioni improprie e locuzioni preposizionali}

davanti «davanti a» 16b.17a.

davanti alle idee «di fronte a» 5 .

dentro della «nella» 21.

fino questo punto 5 .

fino gli anni 5.

fino adesso 21. presso ai 13.

attraverso dell'intuizione 7a. attorno la/ intorno la 11a. inoltre alle $16 \mathrm{~b}$.

nel culmine di «al culmine di» $7 a$.

\section{C ongiunzioni e avverbi}

\section{Congiunzioni subordinative}

mentre che «mentre» $5.11 \mathrm{~b} .15 \mathrm{a} .15 \mathrm{~b} .17 \mathrm{a} .17 \mathrm{~b} . \quad$ tuttavia che «nonostante» 5 .

già que 2 .

già che 18.

di manera 21.

di forma 21.

tanto ... come 22.

\section{Avverbi e locuzioni avverbiali}

più «di più» 3.4 .

a lungo tempo 20.

ogni volta più «sempre più» 11 .

ogni volta di più «sempre di più» 13.

finalmente «alla fine» 19.

propriamente 13 .

giustamente «proprio» 7a.11a.

strictamente «prettamente» $14 a$.

alla stessa volta 11 .

per il contrario 11a.

per contro $6 a$.

per prima volta 2 .

ben contrario «del tutto contrario» 1.

in un principio $17 \mathrm{a}$.

da un primo momento «sin da... » 1 . poco a poco 13.

per ultimo 17a.17b.19.

Anche, diventano «n noltre, diventano» $17 \mathrm{~b}$.

al uguale che 1 .

Anche è considerato « noltre, è considerato» 13 .

no «non» 17b.18.

\section{Sintassi verbale}

\section{Concessive}

sebbene rifiuta 5 .

tuttavia che ... è 5 .

sebbene si perde 9 .

sebbene è 11b.

sebbene ci potevano 16a.

sebbene è spostata $16 \mathrm{~b}$.

benché presenta 20 .

malgrado di essere stato $7 a$.

\section{Gerundio}

riguardando «riguardante» 15 a.

fu adeguando «adeguò» 16a.

dipendendo «dipendenti»16b.

\section{Altri casi}

segnerebbe «avrebbe segnato» $11 b$.

è l'ideale da esser beffato 2 .

è la persona ideale da beffare

espressioni impossibili da capire da essi 8.

impossibili che siano capite da essi/ impossibili da capire 


\section{O rdine di alcune congiunzioni o avverbi}

alla fine Andreuccio soltanto vide due soluzione 1. alla fine Andreuccio vide soltanto

Solo trovano la loro soluzione col suicidio 3.

Trovano la loro soluzione solo col suicidio

il suo amante non dice nulla, soltanto piange 9.

il suo amante non dice nulla, piange soltanto

È anche fortuito il fatto 2 .

È fortuito anche il fatto

l'incontro è anche fortuito 2 .

anchel'incontro è fortuito

Petrarca era anche 12.

Anche Petrarca era

la costruzione ... è anche diversa 19.

anche la costruzione ... è diversa

la $C$ alabria è anche divisa 20.

anche la $\mathrm{C}$ alabria è divisa

anche rifiuta la nozione 5.

rifiuta anche la nozione

anche c'è implicita la nozione 5.

c'è implicita anche la nozione

anche èil tema 9.

è anche il tema

anche si potrà arrivare 13.

si potrà arrivare anche

anche troviamo 19.

troviamo anche

anche può sapere 21 .

può sapere anche

e neanche c'è differenza tra 5 .

e non c'è differenza neanche tra

non sono individuali ma neanche sono 8.

non sono individuali ma non sono neanche 


\section{Lessico}

\section{Prestiti italianizzati}

stamento «classe» 11a.

dettetare «scoprire, individuare» 13. connettare 7a.

\section{Ibridi formali}

risultado 5.

fragmentazione $17 \mathrm{~b}$.

reivindica 5 .

recivono 13.

desviato 22.

devenire 8.

straerne 4.

istoriche 15b.

conflittiva 1.

intervenzione 13.

salvazione 22.

cambio «cambiamento» 20.

estensa «estesa» 20.

paso «passaggio» 17a.

manca «mancanza» 18.

seguitori «seguaci» 13.

esemplarizzarla 1.

evoluziona 5.

evoluzionerà 11 a.

particola 16b.

proposano 4.

emitte 7a.

Tunesia 3.

maiestatico 5.

iustappongono 8.

vantaggiati $11 \mathrm{~b}$.

sollicitati $6 a$.

correspondenti 8.

singulare $15 \mathrm{a}$.

provinente 13.

distinguire 8.

esprimire 13.

simple 17a.

comprova (sost.) «prova» 11b.

Romenia 16b.

\section{Calchi semantici}

darsi «aversi, verificarsi» 6a.6b.16a.17b.21.

soffrirla «subirla» 3.

intorno/entorno «ambiente» $6 \mathrm{~b}$.

cerchio «circolo» $6 a$.

contemplare «considerare» 5. 6b.8.

deriverà «andare verso» $6 \mathrm{~b}$.

sperimentare/ esperim. «subire» 7b.16a.16b.

attiene a «coinvolge» $6 \mathrm{~b}$.

perseguita «perseguitata» 11a.

versare «tradurre» 11a.

allontanato «ontano» $7 a$.

esquisito 《raffinato» 12.

procede «proviene» 20.

resta «è» $11 b$.

affetta a «coinvolge» 20 .

compartono 20.

attuare «agire» 20.22 .

\section{Altri calchi}

il chiamato «il cosiddetto» 19.

fa che «fa si che» $17 \mathrm{~b}$.

non ha niente da vedere 13. non ha niente che vedere 5.

al suo desiderio e piacere 3 .

un felice finale 3. 